\title{
ANDROID DEVICE OPERATED INSULIN PUMP CONNECTED USING BLUETOOTH
}

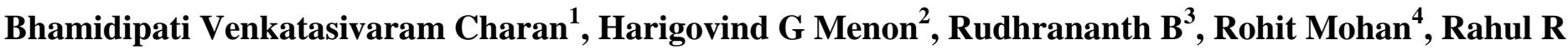

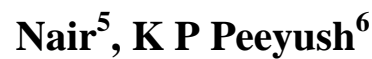 \\ ${ }^{1}$ Student, ECE, Amrita Vishwa Vidyapeetham, Tamilnadu, India \\ ${ }^{2}$ Student, ECE, Amrita Vishwa Vidyapeetham, Tamilnadu, India \\ ${ }^{3}$ Student, ECE, Amrita Vishwa Vidyapeetham, Tamilnadu, India \\ ${ }^{4}$ Student, ECE, Amrita Vishwa Vidyapeetham, Tamilnadu, India \\ ${ }^{5}$ Student, ECE, Amrita Vishwa Vidyapeetham, Tamilnadu, India \\ ${ }^{6}$ Asst. Professor, ECE, Amrita Vishwa Vidyapeetham, Tamilnadu, India
}

\begin{abstract}
Health care today have turned out to be more technology oriented. Diabetes Mellitus is one of the most predominant and common disease prevalent throughout the world. The use of insulin pumps is advocated over manual supplying of insulin to allow precise and accurate delivery of the prescribed volume of insulin over a specified time into the body. There is a drastic increase in the number of people using Android operating system mobile devices. The open sourced nature of android helps in easily programing applications as per the user's requirements. In this paper we describe the design and implementation of an android application for controlling a subcutaneous insulin pump. A user friendly application would be developed which would help the patient administer insulin in both basil and bolus modes. The insulin pump would be programmed using a microcontroller and the connection with the android device would be established using Bluetooth technology. Using android application simplifies the operation of the insulin pump, eliminates manual handling of pump settings and helps in monitoring and tracking every activity of the pump, increasing the efficiency of diagnosis. A well-organized warning system would be incorporated for ensuring that any malfunctions are intercepted and necessary alarms are provided for alerting the user. A history log would also be maintained using android database which could be referred to by the physician.
\end{abstract}

Keywords: Diabetes Mellitus, Bluetooth, Android Device, Insulin pump. $* * *$

\section{INTRODUCTION}

Diabetes has taken a toll on millions of people's life over the past decade. The impact of diabetes has been nothing less than an exponential growth. Diabetes is a disease caused due to elevated blood sugar level in the blood. This happens when the body doesn't produce adequate amount of insulin or respond to it. Diabetes leads to serious metabolic abnormalities and health complications. Some common problems are excess thirst, frequent urination and giddiness.

The insulin pump is a drip-feeding pump used in health cares for administering drugs into a patient's body without causing any discomfort. This kind of medical practice has widely replaced the laborious conventional syringe system in most of the sophisticated health cares. In this paper we have taken up the therapeutic procedure of treating diabetes analyzed it and optimized the insulin pump cutting down the price and complexity.
The main intention of our work is to modify the existing insulin pump making it simpler (hardware-wise), and make the interface sophisticated (software-wise). This is achieved by removing the external peripherals of the micro-controller like buzzers, LCD, LEDs, keypads etc., and introducing an Android device to perform all the above component's operations. An android application is developed to serve this purpose.

Android is currently the most common and popular operating system used in mobiles and tablets. Being an open source platform it is easy for programmers to design and deploy new applications. The proposed system aims at designing such a simple LITE android application available for the patients using the insulin pump. The purpose of the application includes control over the insulin pump for initiating different modes of therapy and alerting the user in case of any malfunction. The application also provides appropriate warning notifications. The link between the android application and the insulin pump is established using Bluetooth technology. The fact that almost everyone has an 
android device in form of a smartphone distance makes us settle down to Bluetooth as the perfect option for data transfer between the pump and the android device.

\section{MOTIVATION FOR THE WORK}

The number of people suffering from diabetes have increased manifold in the past few decades. Diabetes with its never ending demands for care add up to the high octane stress in people's daily life. When not treated with care diabetes causes genetic damage and affects the entire family tree. The complexity and the high cost acts as the gap avoiding the pump from reaching the mass of the public. The main aim of our work is to simplify the existing pump by pertaining its operation to insulin therapy, provide a much simpler interface and make the handling/controlling of the pump easier by using the power of android. This makes the process of insulin infusion more user-friendly and acquirable to the public.

With the development in today's technology it is possible to improve the accuracy, reliability and adaptability of the project to a greater extent. The modified insulin pump allows the subcutaneous infusion of insulin to the patient. The application of the insulin pump can be extended to any therapeutic purpose that demands continuous administration of drugs.

\section{ANDROID APPLICATION ARCHITECTURE}

Android application comprises of different activities. Activities provide the window for the user to interact. An application involves several interrelated activities. Activities comprise of "views" or GUI components like buttons, text box, edit text etc. These views are called when the activity is created. An activity can either be the main activity or it can be called upon from another activity. In both cases the activity has a certain life cycle depending on how it is designated. The activity life cycle includes several states like 'start', 'stop', 'resume', 'pause' and 'destroy'. Start is a state when the activity is about to be visible. The stop is when the activity is no longer visible. The destroy state is when the activity is about to be removed from memory. The pause state is used when an activity is about to take focus from our main activity. Each activity must implement all of the above states. When an activity is called by another activity it enters into pause state saving the current state. When the new activity demands data from the past activity it is passed to the new activity when it is called. This is done to make the data accessible. This can also be achieved by creating a static service that grants access to all activities throughout the life time of the application. In order to fully control the service the necessary functions has to be inherited by the activities.

\section{SYSTEM ARCHITECTURE}

A conceptual view of the system, architecture is given below:

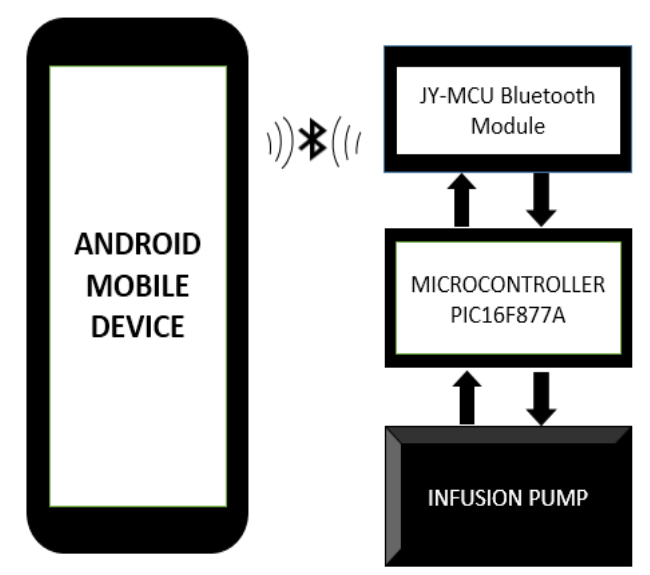

Fig 1 System Architecture

The entire system can be divided into two parts: the android device and the insulin pump. The insulin pump will include microcontroller, syringe based assembly, stepper motor for controlling the motion of the syringe, switches for setting up interrupts and an external Bluetooth for communicating with the android device. The android application will be made using Eclipse Software.

\subsection{Hardware}

The hardware of the system comprises of a microcontroller, an insulin pump, an android device and a Bluetooth module.

The microcontroller used is PIC16F877A. It is a 40 pin IC and has $8 \mathrm{~K}$ flash memory. It can provide upto 14 interrupts. The PIC16 series has five I/O ports for handling data and interrupts. The microcontroller will control the working of the stepper motor for regulating the amount of insulin to be pumped. Bluetooth module is interfaced to the microcontroller through the Universal Asynchronous Data Transfer. The functioning of the interrupts for providing warnings is also the responsibility of the microcontroller.

A syringe based system is used to administer the insulin into the patient. The working of the syringe assembly will be done using the stepper motor which is controlled by the microcontroller.

Switches are provided for getting various interrupts like insulin volume empty, insulin volume full, insulin volume low etc. All the interrupts are provided as external interrupts to the microcontroller.

An external Bluetooth module is provided to establish the data transfer between the microcontroller and the android device. 
The Bluetooth module used in the system is JYMCU Bluetooth module which is powered by a USB B 2.0. The baud rate for the Bluetooth module is $9600 \mathrm{bps}$.

\subsection{Software}

Android is one of the most common operating system used in mobile phones. Being an open sourced platform it allows the design and deployment of android applications easily. Thus android proves to be one of the best platforms for developing an interface for the pump so that it can reach most of the people. A major advantage of android is its simple and quick installation. And being an open sourced platform it allows any change in the interface easily. In the proposed system the entire user interface will be using the android application. The Bluetooth present in the android device is used for transferring data between the android device and the pump.

The android application has been designed and deployed using Eclipse software (Version 3.6). The android SDK provides libraries for interfacing with hardware and to deploy the application. Android applications are coded using JAVA. The source code and the android manifest are written using JAVA code. The layout is made using XML. An activity class, Splash, is extended to create a screen for the background class. The database is made using SQLite. It is designed for less memory and power consumption. Intent class is used to start an activity, service or broadcast from an activity. The android manifest is used for going from one screen to the other as per the user interface.

The PIC programing for the microcontroller is done using MPLAB software in $\mathrm{C}$ language in Hi-Tech compiler. The hex code generated is loaded into the microcontroller through PIC kit 3.

\section{PROCESS FLOW}

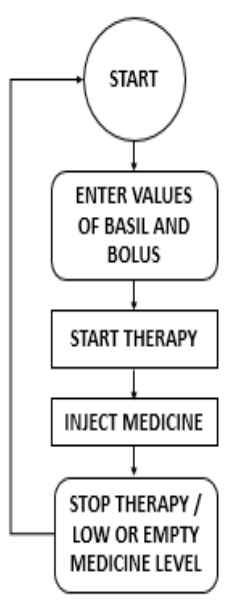

When the application is first launched a menu appears which will allow the user to select between personal details and Therapy. On selecting therapy it will allow the user to select between the basil and bolus mode of insulin administration. On selecting the mode of dosage a subsequent menu appears that lets the user enter the various other details like dosage and time. Once these values are entered it is stored using the SQLite database. When the Start is pressed in the android application it will connect the Bluetooth of the android device with the insulin pump. The dosage and times are communicated to the pump. Once the microcontroller receives the values form the android device it will use these values to control the stepper motor to inject the required dosage of insulin. In case of situations like insulin empty or insulin low interrupts are provided to alert the user through the android application by receiving a signal form the PIC via Bluetooth.

\section{USER INTERFACE}
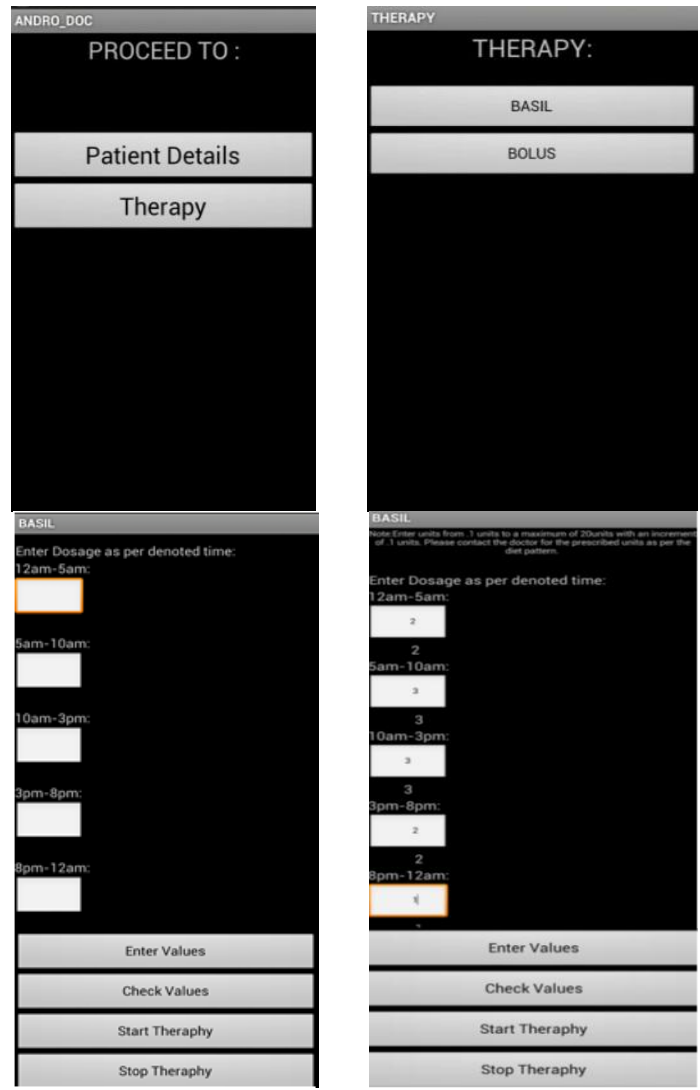

Fig 3 User Interface

The android application is provided with a user friendly interface so that it is easily accessible to everyone. When the application is launched a menu appears with options Therapy and Patient Details. If Patient Details is selected a menu will appear where the user can enter his personal details and his medical background. If Therapy selected a subsequent menu appears with option Basil and Bolus. If Basil is selected a 
subsequent menu appears which will allow the user to enter the dosage at predetermined times. When Enter Values is selected the values get stored in the database. If Start Therapy is selected it will start the administration of the insulin as per the prescribed time. If Stop Therapy is selected it will stop the administration of insulin. If Bolus Mode is selected a menu will appear that will allow the user to enter the dosage and the start and stop time. If Enter Values is selected the values will get stored in the database. If Start Therapy is selected the insulin administration process will begin as per the dosage specified. When Stop Therapy is selected the administration gets stopped.

The amount of dosage of insulin to be administered is specified by the doctor depending on food intake and previous medical history. It will also have a system to warn the user in case of a malfunction in the form of blinking LED and vibratory alerts.

\section{IMPLEMENTATION}

The system comprises of three modules - Insulin Pump, Android application and the Bluetooth module. The first module, the insulin pump, comprises of the design of the insulin pump with the PIC microcontroller, to interface the Bluetooth module with the microcontroller and to provide the necessary interrupts for providing the warnings. The second module comprises of the android application. It interfaces the insulin pump and provides various profiles, warnings and error messages to the user. The third module comprises of link between the insulin pump and the android device provided using the externally provided Bluetooth module.

\subsection{Module 1: Insulin Pump}

Module one comprises of the design of the insulin pump. The insulin pump will include a syringe based system that will be controlled using a stepper motor assembly. The stepper motor will control a retractable system that will help in moving the syringe forward and backward. The system will be designed such that the stepper motor will rotate with a uniform speed. The amount of dosage to be given will be converted to the number of rotation that the pump will have to make to completely pump the required dosage. The number of rotation will be then divided by a factor of ten and will be given at a one minute interval so that the necessary insulin can be administered in the designated time. The pump will have three switches that will be used to control the interrupts for medicine full, low medicine and medicine empty. The PIC microcontroller will be embedded into the pump which will control the stepper motor, the interrupts and the transmission and reception from the Bluetooth module. The Bluetooth module is also separately embedded into the pump for data transfer with the android application.

\subsection{Module 2: Android Application}

Module two comprises of the design and implementation of the android application. The android apps should take the dosage from the user as per the designated set times, Store these values in a database and transfer these values to the insulin pump using Bluetooth. The android app should also provide warnings to the user in case of any malfunction which will be shown in the form of blinking led in the phone and vibratory alerts. The designed application is user friendly and will help the users to easily administer the insulin.

\subsection{Module 3: Bluetooth Link}

Module three comprises of establishing the Bluetooth connection between the android device and the external Bluetooth provided in the pump for data transfer.

\section{CONCLUSIONS}

In this paper, a unique approach has been presented to simplify the current insulin pump. The system comprises recent wireless technology - Bluetooth technology, mobile technology - Android operating system along with commercially available insulin pumps. The advanced, user friendly interface in Android application simplifies the operation of the insulin pump. It eliminates manual handling of pump settings. The detailed, log based database system, SQLite helps in monitoring and tracking every activity of the pump. It generates history report, aiding the diagnosis process and improving the efficiency of the infusion system.

In future, the system can be developed to integrate the patient insulin unit and the hospital server, which will further enhance the efficiency of the diagnosis.

\section{ACKNOWLEDGEMENTS}

The authors would like to thank all the staff of the Department of Electronics and Communication Engineering and Amrita Biomedical Engineering (AMBE), Amrita Vishwa Vidyapeetham, Coimbatore, for their constant support throughout the course of the work.

\section{REFERENCES}

[1] Stavroula G. Mougiakakou, Christos S. Bartsocas, Evangelos Bozas, Nikos Chaniotakis, Dimitra Iliopoulou, Ioannis Kouris, Sotiris Pavlopoulos, Aikaterini Prountzou, Marios Skevofilakas, Alexandre Tsoukalis, Kostas Varotsis, Andrianni Vazeou, Konstantia Zarkogianni, and Konstantina S. Nikita, "SMARTDIAB: A Communication and Information Technology Approach for the Intelligent Monitoring, Management and Follow-up of Type 1 Diabetes Patients," IEEE Transactions on Information Technology In Biomedicine, Vol. 14, No. 3, May 2010. 
[2] Md. Kamrul Hasan, Dr. Nova Ahmed and A. H. M. Saiful Islam," Android Mobile Application: Remote Monitoring of Blood Pressure".

[3] Nivedita Daimiwal, Dipali Ramdasi, Revathi Shriram, and Asmita Wakankar," Wireless Transfusion Supervision and Analysis Using Embedded System," 2010 International Conference on Bioinformatics and Biomedical Technology

[4] Sebastian van Delden and Andrew Whigham," A Bluetooth-based Architecture for Android Communication with an Articulated Robot"

[5] Jianye Liu and Jiankun Yu," Research on Development of Android Applications".

[6] Andrei Drumea and Alexandru Vasile," Infusion pump medical system controlled with modern system on chip devices". ISSE 2006 St. Marienthal, Germany.

[7] David Pager, "The Design and Implementation of an Automatic Computer-Controlled Infusion Pump," Tenth IEEE Symposium on Computer-Based Medical Systems.

[8] Samyuktha Challa, G.Geethakumari and CSN Prasad," Patient Data Viewer: An Android Application for Healthcare.

\section{BIOGRAPHIES}

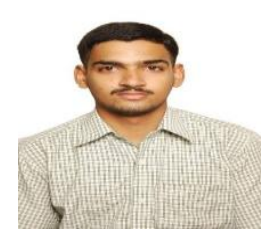

B V S Ramcharan is currently pursuing his Bachelor's degree in Technology in the field of Electronics and Communication Engineering at Amrita Vishwa Vidyapeetham, Coimbatore, India. Ph: +91 8122369344;

Mail: rednair7@gmail.com

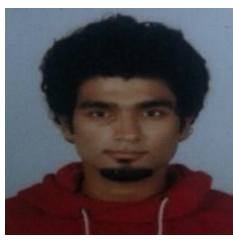

Rohit Mohan is currently pursuing his Bachelor's degree in Technology in the field of Electronics and Communication Engineering at Amrita Vishwa Vidyapeetham, Coimbatore, India. Ph: +91 994086563;

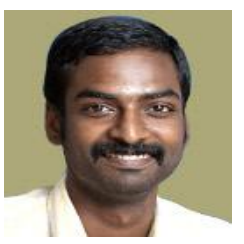

K.P.Peeyush, completed his Masters in Engineering and is currently working as an Assistant Professor in the department of Electronics and Communication Engineering at Amrita Vishwa Vidyapeetham, Coimbatore, India. Ph: +91 9626947031; Mail: peeyushkp@gmail.com

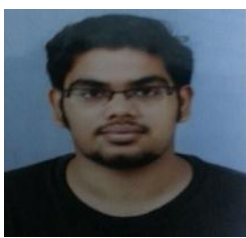

Harigovind Gokul Menon is currently pursuing his Bachelor's degree in Technology in the field of Electronics and Communication Engineering at Amrita Vishwa Vidyapeetham, Coimbatore, India. Ph: $\quad+91 \quad 9567909695 ; \quad$ Mail: harigovindmenon92@gmail.com

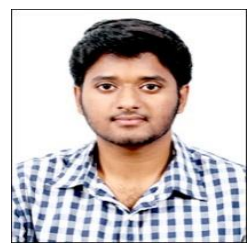

Rudhrananth B is currently pursuing his Bachelor's degree in Technology in the field of Electronics and Communication Engineering at Amrita Vishwa Vidyapeetham, Coimbatore, India. $\mathrm{Ph}$ : rudrananth@gmail.com 8807607402; Mail:

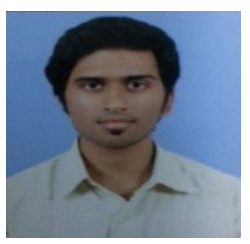

Rahul Ramachandran Nair is currently pursuing his Bachelor's degree in Technology in the field of Electronics and Communication Engineering at Amrita Vishwa Vidyapeetham, Coimbatore, India. Ph: $\quad+91$ 8220583679; $\quad$ Mail: 